\title{
Revisional One Anastomosis Gastric Bypass with a 150-cm Biliopancreatic Limb After Failure of Adjustable Gastric Banding: Mid-Term Outcomes and Comparison Between One- and Two-Stage Approaches
}

\author{
Niccolò Petrucciani ${ }^{1}$ - Francesco Martini ${ }^{2}$ - Marine Benois ${ }^{3} \cdot$ Radwan Kassir $^{3} \cdot$ Hubert Boudrie $^{2}$. \\ Olivier Van Haverbeke ${ }^{2}$. Celine Hamid ${ }^{2}$. Gildas Juglard ${ }^{2} \cdot$ Gianluca Costa $^{4} \cdot$ Tarek Debs $^{5}$. Arnaud Liagre ${ }^{2}$
}

Received: 14 July 2021 / Revised: 8 September 2021 / Accepted: 22 September 2021 / Published online: 5 October 2021

(c) The Author(s) 2021, corrected publication 2022

\begin{abstract}
Purpose Laparoscopic adjustable gastric banding (LAGB) was a common procedure worldwide but associated with a high rate of long-term failure. This study aims to evaluate the safety and effectiveness of conversion to one anastomosis gastric bypass (OAGB) after failed LAGB.

Materials and Methods We undertook a retrospective analysis of a prospectively maintained database in a tertiary referral center for bariatric surgery. All cases of revisional OAGB with a biliopancreatic limb (BPL) of $150 \mathrm{~cm}$ after failed LAGB performed between 2010 and 2016 were analyzed.

Results Overall, 215 patients underwent conversion from LAGB to OAGB. Indication for surgery was primary weight loss (WL) failure in $30.7 \%$ of cases and long-term complications in the remaining patients, with or without associated weight regain. At the time of OAGB, the mean age was $43.2 \pm 10.5$ years and the mean BMI was $42 \pm 6.9$. Overall postoperative morbidity was $13.5 \%$. The postoperative abscess \pm leak rate was $5.9 \%$ in the overall population. Two years after OAGB, 9.7\% of patients were lost to follow-up, \% excess weight loss (EWL) was $88.2 \pm 23.9$, and \% total weight loss (TWL) was $38.7 \pm 9.3$. At 5 years, $16.6 \%$ of patients were lost to follow-up, $\% \mathrm{EWL}$ was $82.4 \pm 25$, and $\% \mathrm{TWL}$ was $36.1 \pm 10$. There was no statistical difference in complication rates or WL results between the one-stage and two-stage approaches.

Conclusion OAGB with a 150-cm BPL represents a safe and effective option after failed LAGB. Both synchronous OAGB and two-step revisional OAGB guarantee satisfying results in terms of postoperative morbidity and WL outcomes.
\end{abstract}

Keywords Bariatric surgery $\cdot$ One anastomosis gastric bypass $\cdot$ Adjustable gastric banding $\cdot$ Complications $\cdot$ Revisional surgery

Key Points

- OAGB represents a potential option for revisional surgery after failure of LAGB.

- Revisional OAGB with a $150-\mathrm{cm}$ limb after LAGB is safe and effective.

- One-stage and two-stage LAGB removal and OAGB have similar results.

Responsible Editor: Jorge Luiz Mello Sampaio

Niccolò Petrucciani

niccolo.petrucciani@uniroma1.it

Extended author information available on the last page of the article

\section{Introduction}

Obesity still represents a global health concern. Bariatric surgery is the most effective therapy for morbid obesity, resulting in sustainable weight loss (WL) and an improvement in obesity-related comorbidities [1, 2]. Laparoscopic adjustable gastric banding (LAGB) was the first minimally invasive bariatric procedure to be widely adopted [3]. In the early 2000s in France, LAGB was the technique of choice in $80 \%$ of patients undergoing bariatric surgery [3-5]. LAGB has been widely practiced in France since 1995, with more than 160,000 procedures performed to date [6]. On a worldwide scale, the number of LAGB procedures represented $24.4 \%$ of the total bariatric procedures in 2003 , although it 
decreased to 5\% in the last International Federation for the Surgery of Obesity and Metabolic Disorders (IFSO) report in 2018 [7, 8].

Even though satisfactory WL results have been reported after LAGB placement and some evidence still supports the choice of a gastric band in selected patients (9), the majority of authors have abandoned LAGB because of high early failure rates and/or inferiority compared to other bariatric procedures [9-11]. Long-term complications include band erosion, band migration, pouch dilatation, intractable nausea, reflux, and port infection [12]. As a result, the adjustable gastric banding (AGB) removal rate has increased over time (about $3-4 \%$ per year), and since 2012, more bands have been removed than have been placed. At present, almost half of the placed bands have been removed [6, 11, 13].

In most cases, patients who have their AGB removed regain weight to presurgery levels. Aarts et al. [14] reported a complete regain of weight at 5 years after LAGB removal in all 21 patients of their series. Rohner et al. [15] reported similar bariatric results in 21 patients at 5 years. Moreover, they found that removal of the banding system alone leads to the deterioration of physical and psychiatric comorbidities, as well as low quality of life scores.

Therefore, a second bariatric procedure should be proposed to all patients when deemed technically feasible and safe. Unfortunately, revisional procedures carry a higher complication rate than their primary counterparts; in particular, the risk of a staple line leak is significantly greater [16].

There is no consensus about which conversional procedure should be offered. The comparison concerns the safety and efficacy of the different procedures. The most common revisional procedures after LAGB failure are the Roux-en-Y gastric bypass (RYGB) and the sleeve gastrectomy (SG) [17]. One anastomosis gastric bypass (OAGB) has been recently proposed by several teams with promising results[18-20]. AGB withdrawal and revisional surgery may be performed in one or two stages. The choice between the two methods is still a matter of debate, such as the optimal time lapse between the two procedures in the case of a two-stage approach.

This study aims to evaluate the safety and effectiveness of conversion to OAGB after failed LAGB in a referral institution for bariatric surgery.

\section{Patients and Methods}

Between May 2010 and December 2016, 215 patients underwent OAGB as a revisional procedure after failed LAGB. Patients were retrieved from a prospectively maintained database of all bariatric procedures performed in our institution. The institutional review board approved the present study.

\section{Preoperative Workup}

Failure of LAGB was defined as insufficient WL (excess weight loss (EWL) $>50 \%$ at 18-month follow-up [21]) and/ or development of long-term complications, including band slippage/displacement, and pouch dilatation. Preoperative workup included upper gastrointestinal (GI) endoscopy, upper GI series, abdominal ultrasound, and clinical, biochemical, nutritional, and psychological assessments. The multidisciplinary obesity board of the institution validated the indication for revisional surgery.

The patients were classified into three groups according to the timing of conversion to OAGB. Patients in the first group (group 1) underwent LAGB removal and synchronous OAGB. In group 2, conversion to OAGB was performed within 12 months from LAGB removal. In group 3, OAGB was performed 12 months or more after LAGB removal.

\section{Surgical Technique}

All revisional procedures after LAGB were performed by an experienced bariatric surgeon with a standardized procedure. OAGB was performed concomitantly with lap-band removal when possible (1-stage procedure) or after a delay (2-stage procedure). The band was always deflated a few weeks before the surgical procedure. At first, the port was liberated and exteriorized from the skin, then the band was identified and dissected from its attachments to the liver; the gastrogastric valve was taken down carefully, and the angle of His was identified. The fibrous capsule surrounding the band was dissected at the level of the His angle to liberate the left crus, and the rest of the scar tissue was not removed.

At this time, the operating surgeon estimated if local conditions allowed a 1-stage procedure or not.

The gastric bypass was performed as previously reported [2]. The lesser sac was entered at the crow's foot, and a long and narrow gastric pouch was fashioned over a 36-Fr calibration tube. The omentum was divided in patients with central obesity to facilitate the ascent of the jejunum. A $150-\mathrm{cm}$ jejunal loop was measured from the Treitz ligament using marked graspers and then an antecolic side-to-side gastrojejunostomy was fashioned using a $60-\mathrm{mm}$ linear stapler.

\section{Postoperative Outcomes and Follow-up}

In the postoperative period, oral liquid intake was resumed at postoperative day 1 , and if liquid intake was tolerated, solid intake was subsequently resumed. Postoperative complications were classified according to the Clavien-Dindo classification [22].

Follow-up was performed at 1 month, 3 months, and 6 months and then every 6 months thereafter and consisted of physical examination and blood tests. The percentage of EWL 
(\%EWL) was calculated using the maximum weight as the initial weight. The $\%$ EWL was calculated as [initial weight - follow-up (FU) weight] / [initial weight -ideal weight] $\times 100$. The ideal weight was set as that equivalent to a BMI of $25 \mathrm{~kg} /$ $\mathrm{m}^{2}$. The percentage of total weight loss (\%TWL) was calculated using the following formula: (weight loss / initial weight $) \times 100$. Residual $\%$ TWL and $\%$ EWL were defined as the WL obtained with the primary treatment (LAGB) at the time of secondary treatment (OAGB). Additional \% TWL after OAGB was defined as (weight loss/initial weight) $\times 100$, using the weight as the time of OAGB as initial weight.

The evolution of obesity-related comorbid conditions was assessed according to the use and discontinuation of medication postoperatively in the instance of diabetes, hypertension, and dyslipidemia. Remission of hypertension was defined as a systolic blood pressure of less than $130 \mathrm{mmHg}$ or a diastolic blood pressure of less than $85 \mathrm{mmHg}$ without the use of antihypertensive drugs. Improvement was defined as a decrease in the quantity or dosage of antihypertensive drugs. Diabetes remission was defined as fasting glucose of less than $5.6 \mathrm{mmol} / \mathrm{L}$ and a glycosylated hemoglobin value of less than $6.5 \%$ without the use of oral hypoglycemic medications or insulin. Improvement was defined as a decrease in the quantity or dosage of oral hypoglycemic medications or insulin. The presence of preoperative sleep apnea syndrome was quantified by sleep studies and postoperative resolution by discontinued use of continuous positive airway pressure masks.

Biliary reflux was defined as the presence of clinical symptoms necessitating treatment, such as heartburn and/or bile vomiting and/or biliary regurgitation, particularly during the night or in dorsal decubitus. Some patients reported mild episodes of hypoglycemia, defined as episodic feelings of faintness between meals (to differentiate from dumping syndrome), associated with glucose values of at least lower than $70 \mathrm{mg} / \mathrm{dL}$ at a glucometer self-assessment.

\section{Statistical Analysis}

Data were expressed as mean \pm standard deviation or median (range) or as numbers and percentages. Comparisons were made using the chi-square test for nominal data and Student's $t$ test for continuous data. A $p$ value $<0.05$ was considered statistically significant. All statistical analyses were performed using SPSS software, version 25.

\section{Results}

\section{Patients' Characteristics}

During the study period, 215 patients underwent conversion from LAGB to OAGB. A flow chart and the timing between AGB removal and OAGB in patients are reported in Fig. 1. Indication for surgery was primary WL failure in $30.7 \%$ of cases and long-term complications from the AGB (band slippage/displacement, pouch dilatation) in the remaining patients, with or without associated weight regain.

Out of the 215 patients included, 195 were females. Before LAGB, the mean weight was $119.7 \pm 19.3$, with a BMI of $44.4 \pm 6.4$. Minimal weight and BMI after LAGB were $85 \pm 18.6$ and $31.8 \pm 6.5$, respectively, with a maximal \%EWL of $66.9 \pm 29$ and a maximal $\% \mathrm{TWL}$ of $28.6 \pm 12.1$. The mean timeframe between AGB removal and $O A G B$ was $14.1 \pm 25.5$ months, and the mean period between LAGB surgery and OAGB was $102.9 \pm 42$ months.

At the time of OAGB, the mean age was $43.2 \pm 10.5$ years (range: $23-68$ ); the mean weight was $113 \pm 21.6$, with a mean BMI of $42 \pm 6.9$. The characteristics of patients are reported in Table 1.

\section{Postoperative Short and Long-Term Complications}

All the OAGBs were performed with a laparoscopic approach. No postoperative mortality occurred. Postoperative short-term and long-term complications are listed in Tables 2 and 3. Overall postoperative morbidity was $13.5 \%$. The postoperative abscess \pm leak rate was $5.9 \%$ in the overall population. All the leaks occurred at the staple line in the area where the band had been placed. Among long-term complications, internal hernias occurred in $3.7 \%$ of patients. Gastro-esophageal reflux that was resistant to medical treatment occurred in $21.3 \%$ of patients and required conversion to RYGB in $4.2 \%$ of cases. Mild episodes of hypoglycemia occurred in $1 \%$ of patients, episodes of diarrhea in $0.5 \%$, and anastomotic ulcers in $0.5 \%$.

One patient died during follow-up from suicide. Compliance with vitamin supplementation was declared by $81.8 \%$ of patients. Iron infusions were administered to $6.5 \%$ of patients during follow-up. No cases of malnutrition requiring hospitalization or OAGB reversal were observed. Results of blood tests are reported in Table 4.

\section{Weight Loss Outcomes (Table 5) and Resolution of Comorbidities}

At 2 years after OAGB, 9.7\% of patients were lost to follow-up, BMI was $28 \pm 5.5 \mathrm{~kg} / \mathrm{m}^{2}, \%$ EWL was $88.2 \pm 23.9$, and $\%$ TWL was $38.7 \pm 9.3$. At 5 years after OAGB, $16.6 \%$ of patients were lost to follow-up, BMI was $29.2 \pm 5.8 \mathrm{~kg} /$ $\mathrm{m}^{2}, \% \mathrm{EWL}$ was $82.4 \pm 25$, and $\% \mathrm{TWL}$ was $36.1 \pm 10$. 
Table 5 reports WL outcomes. Table 6 reports comorbidities and their evolution.

\section{Comparison of AGB Efficacy in Terms of Weight Loss}

Table 7 (supplementary) reports patients' characteristics and outcomes according to the efficacy of the LAGB (defined as \%EWL $>50$ at 18-month follow-up).

\section{Timing of OAGB After AGB Removal}

Table 7 reports patients' characteristics and outcomes according to the timing of OAGB after AGB removal, comparing synchronous OAGB (group 1) versus delayed OAGB within 12 months (group 2), versus delayed OAGB $>12$ months (group 3). Weight and BMI were lower in group 1 . The three groups had similar rates of postoperative complications and comparable WL results at 60-month follow-up. Table 9 (supplementary) reports the rates of postoperative leak and conversion to RYGB because of reflux that was resistant to medical treatment, according to the reason and timing of AGB removal and OAGB fashioning.

\section{Discussion}

This report presents the mid-term outcomes of a cohort of 215 patients who underwent OAGB with a $150-\mathrm{cm}$ BPL as a revisional procedure after failed LAGB. The results suggest that this procedure guarantees satisfying outcomes in terms of postoperative morbidity and WL. Synchronous and twostep revisional OAGB provide comparable results.

LAGB in the past represented a very common bariatric procedure worldwide, because of its technical simplicity and short-term efficacy $[4,23]$. However, recently it has become apparent that LAGB is associated with a remarkable rate of long-term complications, and that AGB removal is frequent, with a rate as high as $40 \%$ at 7-year follow-up [13]. Therefore, revisional surgery after failed LAGB has become common [24, 25].

Two main questions arise for surgeons treating patients with LAGB failure, in whom revisional surgery has been decided after multidisciplinary evaluation. The first question concerns which operation should be recommended. Several options are possible, the most frequent being RYGB and SG [26]. The second question is the timing between AGB removal and the revisional procedure. The surgeries may
Fig. 1 Flow chart of the patients included




be performed in one step or during two different operations with a variable delay $[27,28]$.

OAGB is a more recent bariatric procedure, which has rapidly gained acceptance and diffusion worldwide, and represents $7.6 \%$ of all bariatric operations [7, 29]. It has been recognized by the IFSO as a mainstream bariatric procedure [30], and several studies including thousands of patients have established the efficacy and safety of this procedure in treating obesity and its related comorbidities [31-33]. OAGB has been demonstrated to be effective in the setting of revisional bariatric surgery $[34,35]$.

The present study demonstrates that OAGB is a safe and effective option after the failure of LAGB. In our series of 215 patients, we had a very low rate of severe morbidity and no postoperative deaths. Long-term malnutrition was not observed, and the long-term complication rate was low, the most frequent being internal hernia with a rate of $3.7 \%$ and reflux that is resistant to medical treatment (4.2\%) [36]. Internal hernia after OAGB has been reported as a potential complication, even if it is associated with a low rate of bowel ischemia and a need for intestinal resection [37].
Weight loss outcomes were very encouraging, with a $\%$ EWL of $88.2 \pm 23.9$ and a \% TWL of $38.7 \pm 9.3$ at 2-year follow-up. At 5-year follow-up, BMI was $29.2 \pm 5.8 \mathrm{~kg} /$ $\mathrm{m}^{2}, \%$ EWL was $82.4 \pm 25$, and $\% \mathrm{TWL}$ was $36.1 \pm 10$. We did not find more overall complications or worst results between patients having synchronous AGB removal and OAGB and those having two-step revisional surgery, within 12 months or after 12 months. Even the variation of timing at the second step, within 1 year or after at least 1 year, did not cause statistically significant variations in the postoperative outcomes in our series. However, we emphasize that the lower rate of postoperative leaks was reported in the group of delayed OAGB $>12$ months, and it was as low as $1.7 \%$, versus $8 \%$ in patients with delayed OAGB at $<1$ year and $5.7 \%$ in the synchronous procedure. These results were not statistically significant, but this may be related to the number of the included patients. Among the long-term complications, reflux that is resistant to medical treatment is possible and is usually treated with conversion to RYGB; even for this complication, no significant differences were found between the three groups.
Table 1 Characteristics of the included patients

\begin{tabular}{ll}
\hline$N$ & 215 \\
\hline Age at the time of OAGB & $43.2 \pm 10.5(23-68)$ \\
Sex: females, $n(\%)$ & $195(90.7)$ \\
Weight before LAGB $(\mathrm{kg})$ & $119.7 \pm 19.3(66-233)$ \\
BMI before LAGB $\left(\mathrm{kg} / \mathrm{m}^{2}\right)$ & $44.4 \pm 6.4(33-75)$ \\
Maximal \%EWL after LAGB & $66.9 \pm 29(0-165)$ \\
Maximal \%TWL after LAGB & $28.6 \pm 12.1(0-60.7)$ \\
Minimal weight with AGB $(\mathrm{kg})$ & $85 \pm 18.6(44-150)$ \\
Minimal BMI with AGB (kg/m $\left.{ }^{2}\right)$ & $31.8 \pm 6.5(16.3-56.2)$ \\
Maximal efficacy of AGB in terms of weight loss $(\% \mathrm{EWL}>50), n(\%)$ & $150(69.8)$ \\
Time between LAGB and OAGB (months) & $102.9 \pm 42(0-133)$ \\
Time between AGB removal and OAGB (months) & $14.1 \pm 25.5(22-200)$ \\
Reasons for AGB removal, $n(\%)$ & \\
Intolerance to AGB & $15(6.9)$ \\
Proximal gastric dilatation and weight regain & $115(53.4)$ \\
Perforation & $10(4.6)$ \\
Insufficient weight loss or weight regain & $68(31.6)$ \\
Small bowel obstruction & $1(0.04)$ \\
Others & $4(1.8)$ \\
Age at the time of OAGB & $43.2 \pm 10.5(23-68)$ \\
Weight before OAGB $(\mathrm{kg})$ & $113 \pm 21.6(66-233)$ \\
BMI before OAGB $\left(\mathrm{kg} / \mathrm{m}^{2}\right)$ & $42 \pm 6.9(27-67)$ \\
Residual \%EWL & $10.5 \pm 29.6(-124-83)$ \\
Residual \%TWL & $5.4 \pm 11.8(-30.1-39.2)$ \\
Patients with higher weight at OAGB than at LAGB, $n(\%)$ & $63(29.3)$ \\
Efficacy of AGB in terms of weight loss before OAGB $(\% \mathrm{EWL}>50), n(\%)$ & $16(7.4)$ \\
\hline & \\
\hline
\end{tabular}

Data are presented as mean $\pm \mathrm{SD}$ and range for continuous variables, as the absolute number and percentages for categorical variables 
Table 2 Postoperative early complications after revisional OAGB for failed LAGB

\begin{tabular}{llr}
\hline Short-term complications & $N(\%)$ & Clavien-Dindo grading \\
\hline Death & $0(0)$ & \\
Perianastomotic abscess \pm leak & $12(5.6)$ & Grade $2(n=1)$ \\
& & Grade 3a $(n=6)$ \\
& & Grade 3b $(n=4)$ \\
& & Grade $4(n=1)$ \\
Pneumonia/atelectasis & $5(2.3)$ & Grade 2 $(n=5)$ \\
Small bowel perforation & $1(0.5)$ & Grade 3b $(n=1)$ \\
Anastomotic inflammation without abscess & $1(0.5)$ & Grade 2 $(n=1)$ \\
Phlebitis & $1(0.5)$ & Grade 2 $(n=1)$ \\
Myocardial infarction & $1(0.5)$ & Grade 2 $(n=1)$ \\
Pneumothorax & $1(0.5)$ & Grade 3a $(n=1)$ \\
GI bleeding without the need of transfusion & $1(0.5)$ & Grade $1(n=1)$ \\
Bleeding from the drain without the need of transfusion & $4(1.9)$ & Grade $1(n=4)$ \\
Anastomotic stenosis & $2(0.9)$ & Grade 3a $(n=2)$ \\
Total & $29(13.5)$ & \\
\hline
\end{tabular}

Table 3 Postoperative late complications and additional procedures after revisional OAGB for failed LAGB

\begin{tabular}{ll}
\hline Complication/additional procedure & $N(\%)$ \\
\hline Reflux & $46(21.4)$ \\
Medical treatment & $37(17.2)$ \\
Surgical treatment & $9(4.2)$ \\
Internal hernia & $8(3.7)$ \\
Anastomotic ulcer & $4(1.9)$ \\
Medical treatment & $3(1.4)$ \\
Surgical treatment & $1(0.5)$ \\
Insufficient weight loss & $1(0.5)$ \\
Treated with banding of the gastroplasty & \\
Invalidating diarrhea & $13(6.0)$ \\
Medical treatment & $12(5.6)$ \\
Surgical treatment & $1(0.5)$ \\
Hypoglycemia & $2(0.9)$ \\
Medical treatment & $2(0.9)$ \\
Surgical treatment & $0(0)$ \\
Intestinal invagination & $1(0.5)$ \\
Incisional hernia & $4(1.9)$ \\
Suicide & $1(0.5)$ \\
Cholecystectomy & $17(7.9)$ \\
Laparoscopic exploration for abdominal pain & $2(0.9)$ \\
\hline
\end{tabular}

The decision to convert the OAGB for reflux resistant to medical treatment is based not only on esophageal impedance PH testing [38], but on a complete assessment also including computed tomography with oral contrast ingestion and upper gastrointestinal endoscopy. In our experience, conversion to RYGB was effective in $>90 \%$ of patients [36]. Hiatal hernia should be systematically searched for as it may be responsible for the reflux, and in these cases, surgical treatment of the hiatal hernia may permit the remission of the reflux.

In our experience, OAGB after AGB is associated with a higher rate of leaks and transformation to RYGB for reflux, compared to OAGB as a primary procedure [32]. Even if no significant differences were found, a trend for lower rates of leaks was found in patients with OAGB fashioned more than 12 months after AGB removal, whereas similar leak rates were found in patients with synchronous procedures or AGB removal and OAGB within 12 months.

Our results show that OAGB as a revisional procedure after LAGB has a very satisfying profile in terms of safety and efficacy. The advantages of OAGB as a revisional procedure after LAGB are that the scar tissue due to the band does not need to be completely removed because the anastomosis is performed much lower. The dissection is done through the omental bursa, and the preparation of the gastrojejunal anastomosis is done in fresh and "healthy tissue." It should be noted, as reported previously, that our OAGB technique consists in the fashioning of the anastomosis at $150 \mathrm{~cm}$ from the Treitz ligament [39]. Strengths of the present study are the large number of included patients (to our knowledge, it represents the largest singleinstitution series), the standardized surgical technique, and the remarkable 5-year follow-up rate.

Several authors have studied the outcomes of revisional procedures after failed LAGB. The first studies reported conversion to RYGB [40] or compared the outcomes of revisional SG versus RYGB, with no significant differences in postoperative outcomes [26, 41].

Al-Kurd et al. [42] compared RYGB after failed LAGB versus primary RYGB, including 161 patients in both groups. They showed no differences in short-term and long-term 
Table 4 Results of blood tests of patients who underwent OAGB as a revisional procedure after failed LAGB

\begin{tabular}{llrr}
\hline Variable & Preoperative, \% abnormal & 12 months, \% abnormal & 24 months, \% abnormal \\
\hline Hemoglobin $(\mathrm{g} / \mathrm{dL})$ & $13.5 \pm 1.1(N=205), 5.8 \%$ & $13.3 \pm 1.2(N=67), 7.5 \%$ & $13.2 \pm 1.3(N=47), 9 \%$ \\
Albumin $(\mathrm{g} / \mathrm{dL})$ & $38.3 \pm 3.7(N=144), 14.5 \%$ & $40.9 \pm 3.7(N=63), 7 \%$ & $39.9 \% \pm 3.6(N=42), 5 \%$ \\
Ferritin $(\mu \mathrm{g} / \mathrm{L})$ & $145 \pm 126(N=145), 6.2 \%$ & $84.9 \pm 80.9(N=64), 9.4 \%$ & $71.6 \pm 56.1(N=42), 4.8 \%$ \\
Prealbumin $(\mathrm{g} / \mathrm{L})$ & $0.24 \pm 0.05(N=108), 14.8 \%$ & $0.22 \pm 0.05(N=37), 24.4 \%$ & $0.23 \pm 0.05(N=32), 11.9 \%$ \\
Vitamin A $(\mu \mathrm{mol} / \mathrm{L})$ & $2.23 \pm 0.7(N=91), 17.5 \%$ & $1.46 \pm 0.62(N=59), 88 \%$ & $1.75 \pm 0.59(N=37), 62.2 \%$ \\
Vitamin $\mathrm{B}_{1}(\mathrm{nmol} / \mathrm{L})$ & $\mathrm{NR}$ & $148.3 \pm 33.8(N=22), 0 \%$ & $153.4 \pm 44.5(N=7), 14.3 \%$ \\
Vitamin $\mathrm{B}_{9}(\mathrm{ng} / \mathrm{L})$ & $16 \pm 5.5(N=141), 2.8 \%$ & $20.3 \pm 14.5(N=63), 19.1 \%$ & $21.2 \pm 14.2(N=40), 22.5 \%$ \\
Vitamin $\mathrm{B}_{12}(\mathrm{pmol} / \mathrm{L})$ & $319 \pm 107(N=145), 2 \%$ & $313 \pm 140.3(N=60), 3.4 \%$ & $322 \pm 146.6(N=43), 0 \%$ \\
Vitamin $\mathrm{D}(\mathrm{ng} / \mathrm{mL})$ & $42 \pm 20(N=144), 87.5 \%$ & $69.5 \pm 27.2(N=65), 60 \%$ & $70.5 \pm 26.4(N=43), 58.2 \%$ \\
Parathyroid hormone $(\mathrm{pg} / \mathrm{mL})$ & $\mathrm{NR}$ & $61.4 \pm 29.2(N=33), 60.4 \%$ & $56.1 \pm 24.7(N=31), 61.3 \%$ \\
Calcium $(\mathrm{mmol} / \mathrm{L})$ & $2.35 \pm 0.1(N=151), 0.6 \%$ & $2.33 \pm 0.1(N=66), 3 \%$ & $2.29 \pm 0.09(N=44), 4.6 \%$ \\
\hline
\end{tabular}

postoperative morbidity rates $7.5 \%$ in the revisional group versus $11.8 \%$ in the primary RYGB, non-significant) but

Table 5 Weight loss results of patients who underwent revisional OAGB after LAGB

\begin{tabular}{|c|c|}
\hline Variable & Mean \pm SD or $N(\%)$ \\
\hline \multicolumn{2}{|c|}{ At 12-month follow-up after $O A G B(n=215)$} \\
\hline Weight & $80.4 \pm 17.1(50-168)$ \\
\hline BMI & $29.8 \pm 5.6(18-53)$ \\
\hline Lost to follow-up & $15(6.9 \%)$ \\
\hline$\% \mathrm{EWL}$ & $78.8 \pm 21.7(19-143)$ \\
\hline$\% \mathrm{TWL}$ & $34.3 \pm 8.8(9-56.6)$ \\
\hline Additional \% TWL & $28.4 \pm 8.9(6.2-50.5)$ \\
\hline \multicolumn{2}{|c|}{ At 24-month follow-up after $O A G B(n=215)$} \\
\hline Weight & $75.2 \pm 17.3(48-175)$ \\
\hline BMI & $28 \pm 5.5(17-51)$ \\
\hline Lost to follow-up & $21(9.7 \%)$ \\
\hline$\%$ EWL & $88.2 \pm 23.9(28-158)$ \\
\hline$\% \mathrm{TWL}$ & $38.7 \pm 9.3(10-62.9)$ \\
\hline Additional \% TWL & $33.2 \pm 9.7(6.2-58.4)$ \\
\hline \multicolumn{2}{|c|}{ At 60-month follow-up after $O A G B(n=168)$} \\
\hline Weight & $78.2 \pm 16.9(51-130)$ \\
\hline BMI & $29.2 \pm 5.8(18-52)$ \\
\hline Lost to follow-up & $28(16.6 \%)$ \\
\hline$\%$ EWL & $82.4 \pm 25(29-158)$ \\
\hline$\% \mathrm{TWL}$ & $36.1 \pm 10(12.9-61.1)$ \\
\hline Additional \% TWL & $30.5 \pm 11(-4.3-52.35)$ \\
\hline \multicolumn{2}{|c|}{ At $>$ 84-month follow-up after $O A G B(n=110)$} \\
\hline Weight & $79.1 \pm 17.8(51-130)$ \\
\hline BMI & $29.7 \pm 6.4(18-54)$ \\
\hline Lost to follow-up & $31(28.1 \%)$ \\
\hline$\%$ EWL & $80.2 \pm 28.3(28-158)$ \\
\hline$\% \mathrm{TWL}$ & $33.9 \pm 10.2(9.5-56.2)$ \\
\hline Additional \%TWL & $27.7 \pm 10.9(-2.1$ to 52.5$)$ \\
\hline
\end{tabular}

Data are presented as mean \pm standard deviation (range) or as number (percentage) better WL results for primary RYGB (61.5\% vs. $75.5 \%$ of EWL, respectively, with 6-month follow-up attained in $78 \%$ of the patients).

Poublon et al. [43] recently published an interesting and well-conducted study, comparing OAGB versus RYGB after the failure of LAGB or SG. They included 306 revisional RYGB and 185 revisional OAGB. Previous bariatric surgery consisted of SG in $28.5 \%$ of patients and LAGB in the remaining cases. Intra-abdominal complications (leakage, bleeding, intra-abdominal abscess, and perforation) were significantly less frequent after revisional OAGB $(1.1 \%$ vs. $4.9 \%, p=0.025)$. However, revisional surgery for biliary reflux $(5.4 \%$ vs. $0.3 \%, p<0.001)$ was more prevalent in the OAGB group. On the other hand, surgical intervention for internal herniation $(0.0 \%$ vs. $4.9 \%, p=0.002)$ was more common in the RYGB group. Concerning WL results, OAGB guaranteed better outcomes, with larger \% TWL at 12 months (mean $24.1 \pm 9.8$ vs. $21.9 \pm 9.7, p=0.023$ ) and 24 months (mean $23.9 \pm 11.7$ vs. $20.5 \pm 11.2, p=0.023$ ) of follow-up. A greater \% excess BMI loss (EBMIL) was also reported for OAGB [43].

Chansaenroj et al. [44] published concordant results. The authors included 53 patients undergoing OAGB [24], SG [16], and RYGB [45] after failed LAGB. In this study, patients who underwent revisional OAGB had better WL at 1- and 2-year follow-ups. However, no significant differences in \%EWL were reported. Similarly, in a series by Almalki et al. [46] that included 116 patients who underwent OAGB (81) or RYGB (35) after a failed restrictive bariatric procedure, OAGB was associated with better WL results. At 1-year follow-up, \%EWL was $76.8 \%$ in the OAGB group versus $32.9 \%$ in the RYGB group. The major morbidity rate was $10 \%$ in the overall population without significant differences between the two groups.

Data about the long-term results of conversion from LAGB to OAGB are lacking. Only Bruzzi et al. [47] report data of 30 patients who underwent OAGB after failed 
Table 6 Evolution of comorbidities after revisional OAGB

\begin{tabular}{|c|c|c|c|}
\hline Comorbidity & Before OAGB & At last follow-up & Regression (\%) \\
\hline Arterial hypertension & $14.4 \%(31 / 215)$ & $\begin{array}{r}\text { Lost to follow-up }=2 \\
\text { Resolution }=21\end{array}$ & 72 \\
\hline Diabetes & $7 \%(15 / 215)$ & $\begin{aligned} \text { Lost to follow-up } & =5 \\
\text { Resolution } & =9\end{aligned}$ & 90 \\
\hline OSAS & $4.1 \%(9 / 215)$ & $\begin{aligned} \text { Lost to follow-up } & =0 \\
\text { Resolution } & =7\end{aligned}$ & 77 \\
\hline Dyslipidemia & $7 \%(15 / 215)$ & $\begin{array}{r}\text { Lost to follow-up }=1 \\
\text { Resolution }=14\end{array}$ & 100 \\
\hline
\end{tabular}

restrictive procedures. In this series, the major complication rate was $10 \%$ and two patients required conversion from revisional OAGB to RYGB for resistant reflux. At 5 years, the $\%$ EBMIL was $66 \%$. The results of this series are satisfying and comparable with the long-term results of our present series, showing the long-term efficacy of revisional OAGB.

Parmar et al. [48] systematically reviewed the literature retrieving 17 studies including 1075 cases of OAGB after failed LAGB, SG, vertical banded gastroplasty, and gastric plication. Patients had a median limb length of $200 \mathrm{~cm}$, which differs from our technique [39]. The leak rate of this series was $1.54 \%$, and the marginal ulcer rate was $2.44 \%$. Mortality was $0.3 \%$, and the $\%$ EWL at 1 year and 2 years was $65.2 \%$ and $68.5 \%$, respectively. The mean follow-up was 2.44 years.

AGB removal and conversional surgery may be performed concomitantly or in a staged fashion. The more appropriate approach remains a topic of discussion. Surgeons in favor of a single stage argue that this method requires fewer total surgeries and avoids weight regain, which constantly follows a period of non-restriction [49]. Those in favor of a two-stage approach claim that the interval between procedures allows for inflammation to be reduced and for vascularization to be improved at the fibrotic portion of the stomach where the band was placed, therefore limiting the risk of staple line leak [50].

We did not find more overall complications or worst results between patients having synchronous and two-step revisional surgery. Even the variation of timing of the second step, within 1 year or after at least 1 year, did not cause statistically significant major variations in the postoperative outcomes in our series. However, we emphasize that a lower rate of postoperative leaks was reported in the group with the OAGB delayed for $>12$ months, and it was as low as $1.7 \%$, versus $8 \%$ in patients with OAGB delayed for $<1$ year and $5.7 \%$ in the synchronous procedure. These results were not statistically significant, but this may be related to the high number of patients included. Among long-term complications, reflux that was resistant to medical treatment is possible and is usually treated with conversion to RYGB; even for this complication, no significant differences were found between the three groups.

In our experience, OAGB after AGB is associated with a higher rate of leaks and transformation to RYGB for reflux, compared to OAGB as a primary procedure. Even if no significant differences were found, a trend for a lower rate of leaks was found in patients with OAGB fashioned more than 12 months after AGB removal, whereas similar rates of leaks were found in patients with synchronous procedures or AGB removal and OAGB within 12 months.

Lessing et al. [51] reported data on 57 patients undergoing synchronous (41 patients) or two-step conversion from LAGB to OAGB. The complication rate was $15.7 \%$, and one postoperative death occurred. The mean $\%$ EWL was $64.5 \%$ at 1-year follow-up, and no differences were reported between synchronous and two-step cases. Schäfer et al. [28] analyzed the timing of conversion from LAGB to RYGB in a series of 165 patients, reporting a major complication rate of $15.3 \%$ for one-stage surgeries versus $16.9 \%$ for two-step procedures and no significant differences.

Our results show that OAGB as a revisional procedure after LAGB has a very satisfying profile in terms of safety and efficacy. It should be emphasized that our OAGB technique fashions the anastomosis at $150 \mathrm{~cm}$ from the Treitz ligament. Strengths of the present study are the large number of patients included (to our knowledge, it represents the largest singleinstitution series) and the standardized surgical technique.

\section{Limits}

The present study has several limitations including its singlecenter and retrospective design. However, the number of patients is remarkable considering the single-center design of the study. The 5-year follow-up rate of more than $80 \%$ may be considered adequate in the context of bariatric literature where very few published studies have an FU of $70 \%$ or more [52].

\section{Conclusion}

OAGB with a biliopancreatic limb of $150 \mathrm{~cm}$ represents a safe and effective option after failed LAGB. Both synchronous OAGB and two-step revisional OAGB guarantee 
Table 7 Patients' characteristics and outcomes according to the timing of OAGB after AGB removal

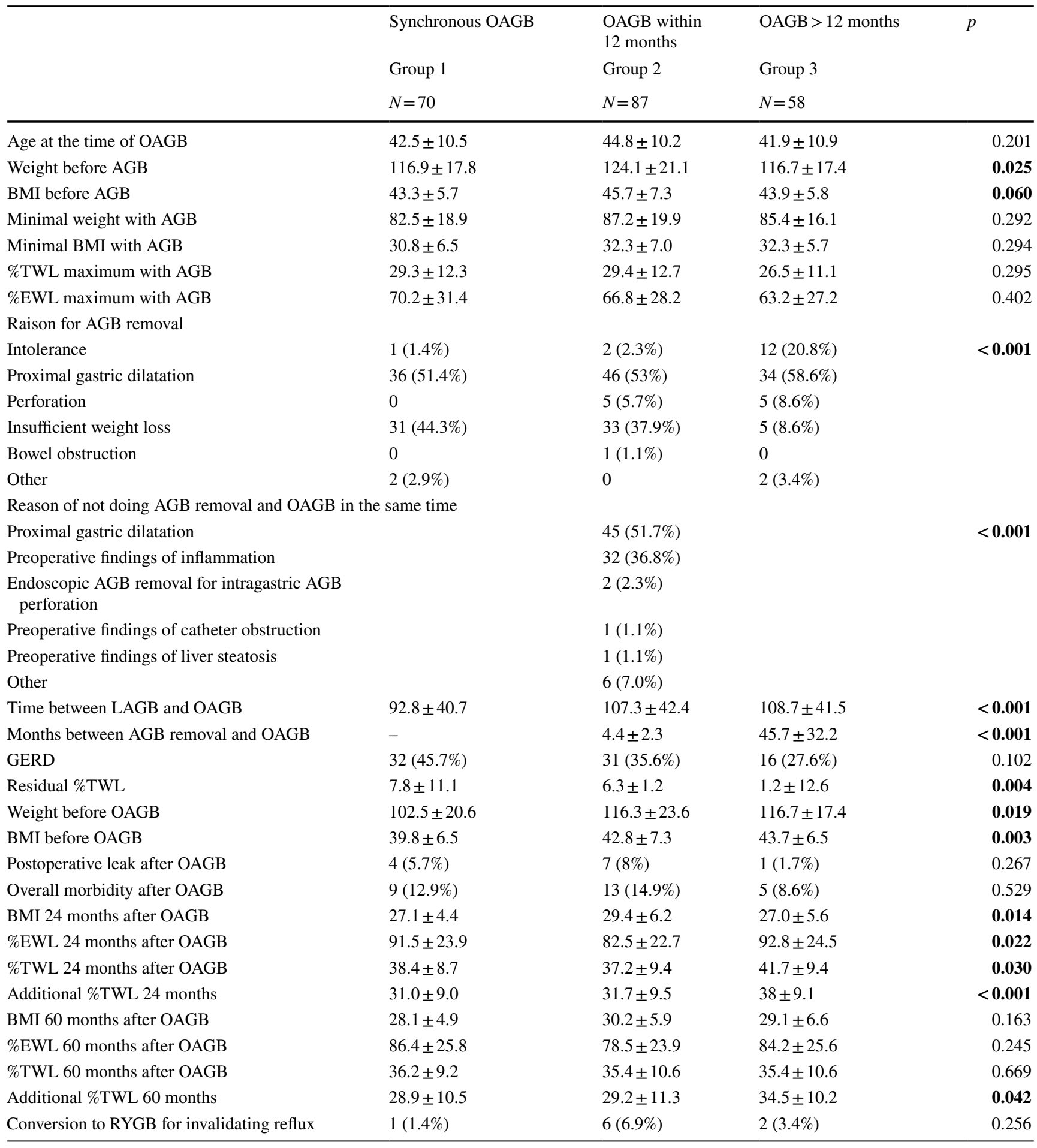

Preoperative and postoperative variables are compared between patients undergoing synchronous AGB removal and OAGB and delayed OAGB after AGB removal. Data are presented as mean $\pm \mathrm{SD}$ for continuous variables, as absolute number and percentages for categorical variables (one-way ANOVA). Significant values are reported in bold 
satisfying results in terms of postoperative morbidity and WL outcomes.

Supplementary Information The online version contains supplementary material available at https://doi.org/10.1007/s11695-021-05728-9.

Funding Open access funding provided by Università degli Studi di Roma La Sapienza within the CRUI-CARE Agreement.

\section{Declarations}

Ethics Approval and Consent to Participate All procedures performed in studies involving human participants were in accordance with the ethical standards of the institutional and/or national research committee and with the 1964 Helsinki Declaration and its later amendments or comparable ethical standards. Informed consent was obtained from all individual participants included in the study.

Conflict of Interest The authors declare no competing interests.

Open Access This article is licensed under a Creative Commons Attribution 4.0 International License, which permits use, sharing, adaptation, distribution and reproduction in any medium or format, as long as you give appropriate credit to the original author(s) and the source, provide a link to the Creative Commons licence, and indicate if changes were made. The images or other third party material in this article are included in the article's Creative Commons licence, unless indicated otherwise in a credit line to the material. If material is not included in the article's Creative Commons licence and your intended use is not permitted by statutory regulation or exceeds the permitted use, you will need to obtain permission directly from the copyright holder. To view a copy of this licence, visit http://creativecommons.org/licenses/by/4.0/.

\section{References}

1. Buchwald H, Consensus Conference Panel. Consensus conference statement bariatric surgery for morbid obesity: health implications for patients, health professionals, and third-party payers. Surg Obes Relat Dis. 2005;1:371-81.

2. Colquitt JL, Pickett K, Loveman E, et al. Surgery for weight loss in adults. Cochrane Database Syst Rev. 2014;8:CD003641.

3. Belachew M, Legrand MJ, Defechereux TH, et al. Laparoscopic adjustable silicone gastric banding in the treatment of morbid obesity A preliminary report. Surg Endosc. 1994;8:1354-6.

4. Debs T, Petrucciani N, Kassir R, et al. Trends of bariatric surgery in France during the last 10 years: analysis of 267,466 procedures from 2005-2014. Surg Obes Relat Dis. 2016;12:1602-9.

5. Buchwald H, Williams SE. Bariatric surgery worldwide 2003. Obes Surg. 2004;14:1157-64.

6. ATIH (Agence Technique de l'information sur l'hospitalisation). PMSI Database. [Internet]. 2020. Available from: http://www. scansante.fr/applications/statistiques-activite-MCO-par-diagn ostique-et-actes?secteur $=\mathrm{MCO}$

7. Welbourn R, Hollyman M, Kinsman R, et al. Bariatric surgery worldwide: baseline demographic description and one-year outcomes from the Fourth IFSO Global Registry Report 2018. Obes Surg. 2019;29:782-95.

8. Angrisani L, Santonicola A, Iovino P, et al. Bariatric surgery worldwide 2013. Obes Surg. 2015;25:1822-32.
9. Suter M, Calmes JM, Paroz A, et al. A 10-year experience with laparoscopic gastric banding for morbid obesity: high long-term complication and failure rates. Obes Surg. 2006;16:829-35.

10. Himpens J, Cadière G-B, Bazi M, et al. Long-term outcomes of laparoscopic adjustable gastric banding. Arch Surg Chic Ill. 1960;2011(146):802-7.

11. Carandina S, Tabbara M, Galiay L, et al. Long-term outcomes of the laparoscopic adjustable gastric banding: weight loss and removal rate A single-center experience on 301 patients with a minimum follow-up of 10 years. Obes Surg. 2017;27:889-95.

12. Zadeh J, Le C, Ben-David K. Safety of adjustable gastric band conversion surgery: a systematic review and meta-analysis of the leak rate in 1- and 2-stage procedures. Surg Obes Relat Dis. 2020;16:437-44.

13. Lazzati A, De Antonio M, Paolino L, et al. Natural history of adjustable gastric banding: lifespan and revisional rate: a nationwide study on administrative data on 53,000 Patients. Ann Surg. 2017;265:439-45.

14. Aarts EO, Dogan K, Koehestanie P, et al. What happens after gastric band removal without additional bariatric surgery? Surg Obes Relat Dis. 2014;10:1092-6.

15. Rohner A, Widmer JD, Klasen J, et al. Long-term outcomes of gastric band removal without additional bariatric surgery. Surg Obes Relat Dis. 2017;13:261-6.

16. Stroh C, Benedix D, Weiner R, et al. Is a one-step sleeve gastrectomy indicated as a revision procedure after gastric banding? Data analysis from a quality assurance study of the surgical treatment of obesity in Germany. Obes Surg. 2014;24:9-14.

17. Mahawar KK, Nimeri A, Adamo M, et al. Practices concerning revisional bariatric surgery: a survey of 460 Surgeons. Obes Surg. 2018;28:2650-60.

18. Musella M, Bruni V, Greco F, et al. Conversion from laparoscopic adjustable gastric banding (LAGB) and laparoscopic sleeve gastrectomy (LSG) to one anastomosis gastric bypass (OAGB): preliminary data from a multicenter retrospective study. Surg Obes Relat Dis. 2019;15:1332-9.

19. O'Brien PE, Hindle A, Brennan L, et al. Long-term outcomes after bariatric surgery: a systematic review and meta-analysis of weight loss at 10 or more years for all bariatric procedures and a single-centre review of 20-year outcomes after adjustable gastric banding. Obes Surg. 2019;29:3-14.

20. Pujol Rafols J, Al Abbas AI, Devriendt S, et al. Roux-en-Y gastric bypass, sleeve gastrectomy, or one anastomosis gastric bypass as rescue therapy after failed adjustable gastric banding: a multicenter comparative study. Surg Obes Relat Dis. 2018;14:1659-66.

21. Mann JP, Jakes AD, Hayden JD, et al. Systematic review of definitions of failure in revisional bariatric surgery. Obes Surg. 2015;25:571-4.

22. Clavien PA, Barkun J, de Oliveira ML, et al. The Clavien-Dindo classification of surgical complications: five-year experience. Ann Surg. 2009;250:187-96.

23. Angrisani L, Santonicola A, Iovino P, et al. IFSO Worldwide Survey 2016: primary, endoluminal, and revisional procedures. Obes Surg. 2018;28:3783-94.

24. Mognol P, Chosidow D, Marmuse J-P. Laparoscopic conversion of laparoscopic gastric banding to Roux-en-Y gastric bypass: a review of 70 patients. Obes Surg. 2004;14:1349-53.

25. Gagner M, Gumbs AA. Gastric banding: conversion to sleeve, bypass, or DS. Surg Endosc. 2007;21:1931-5.

26. Moon RC, Teixeira AF, Jawad MA. Conversion of failed laparoscopic adjustable gastric banding: sleeve gastrectomy or Roux-enY gastric bypass? Surg Obes Relat Dis. 2013;9:901-7.

27. Van Nieuwenhove Y, Ceelen W, Van Renterghem K, et al. Conversion from band to bypass in two steps reduces the risk for anastomotic strictures. Obes Surg. 2011;21:501-5. 
28. Schäfer A, Gehwolf P, Umlauft J, et al. Revisional gastric bypass after failed adjustable gastric banding-one-stage or two-stage procedure? Obes Surg. 2019;29:943-8.

29. Parmar CD, Mahawar KK. One anastomosis (mini) gastric bypass is now an established bariatric procedure: a systematic review of 12,807 patients. Obes Surg. 2018;28:2956-67.

30. De Luca M, Tie T, Ooi G, et al. Mini gastric bypass-one anastomosis gastric bypass (MGB-OAGB)-IFSO position statement. Obes Surg. 2018;28:1188-206.

31. Carbajo MA, Luque-de-León E, Jiménez JM, et al. Laparoscopic one-anastomosis gastric bypass: technique, results, and long-term follow-up in 1200 patients. Obes Surg. 2017;27:1153-67.

32. Liagre A, Queralto M, Juglard G, et al. Multidisciplinary management of leaks after one-anastomosis gastric bypass in a single-center series of 2780 consecutive patients. Obes Surg. 2019;29:1452-61.

33. Musella M, Apers J, Rheinwalt K, et al. Efficacy of Bariatric surgery in type 2 diabetes mellitus remission: the role of mini gastric bypass/one anastomosis gastric bypass and sleeve gastrectomy at 1 year of follow-up. A European survey Obes Surg. 2016;26:933-40.

34. Debs T, Petrucciani N, Kassir R, et al. Laparoscopic conversion of sleeve gastrectomy to one anastomosis gastric bypass for weight loss failure: mid-term results. Obes Surg. 2020;30:2259-65.

35. Chiappetta S, Stier C, Scheffel O, et al. Mini/one anastomosis gastric bypass versus Roux-en-Y gastric bypass as a second step procedure after sleeve gastrectomy-a retrospective cohort study. Obes Surg. 2019;29:819-27.

36. Kassir R, Petrucciani N, Debs T, et al. Conversion of one anastomosis gastric bypass (OAGB) to Roux-en-Y gastric bypass (RYGB) for biliary reflux resistant to medical treatment: lessons learned from a retrospective series of 2780 consecutive patients undergoing OAGB. Obes Surg. 2020;30:2093-8.

37. Petrucciani N, Martini F, Kassir R, et al. Internal hernia after one anastomosis gastric bypass (OAGB): lessons learned from a retrospective series of 3368 consecutive patients undergoing OAGB with a biliopancreatic limb of $150 \mathrm{~cm}$. Obes Surg. 2021;31:2537-44.

38. Nehmeh WA, Baratte C, Rives-Lange C, et al. Acid reflux is common in patients with gastroesophageal reflux disease after oneanastomosis gastric bypass. Obes Surg. 2021. https://doi.org/10. 1007/s11695-021-05542-3

39. Liagre A, Debs T, Kassir R, et al. One anastomosis gastric bypass with a biliopancreatic limb of $150 \mathrm{~cm}$ : weight loss, nutritional outcomes, endoscopic results, and quality of life at 8-year followup. Obes Surg. 2020;30:4206-17.

40. van Wageningen B, Berends FJ, Van Ramshorst B, et al. Revision of failed laparoscopic adjustable gastric banding to Roux-en-Y gastric bypass. Obes Surg. 2006;16:137-41.
41. Liu K-H, Diana M, Vix M, et al. Revisional surgery after failed adjustable gastric banding: institutional experience with 90 consecutive cases. Surg Endosc. 2013;27:4044-8.

42. Al-Kurd A, Grinbaum R, Abubeih A, et al. Conversion of laparoscopic adjustable gastric banding to gastric bypass: a comparison to primary gastric bypass. Obes Surg. 2018;28:1519-25.

43. Poublon N, Chidi I, Bethlehem M, et al. One anastomosis gastric bypass vs. Roux-en-Y gastric bypass, remedy for insufficient weight loss and weight regain after failed restrictive bariatric surgery. Obes Surg. 2020;30:3287-94.

44. Chansaenroj P, Aung L, Lee W-J, et al. Revision procedures after failed adjustable gastric banding: comparison of efficacy and safety. Obes Surg. 2017;27:2861-7.

45. O'Brien PE, MacDonald L, Anderson M, et al. Long-term outcomes after bariatric surgery: fifteen-year follow-up of adjustable gastric banding and a systematic review of the bariatric surgical literature. Ann Surg. 2013;257:87-94.

46. Almalki OM, Lee W-J, Chen J-C, et al. Revisional gastric bypass for failed restrictive procedures: comparison of singleanastomosis (mini-) and Roux-en-Y gastric bypass. Obes Surg. 2018;28(4):970-5.

47. Bruzzi M, Voron T, Zinzindohoue F, et al. Revisional single-anastomosis gastric bypass for a failed restrictive procedure: 5 -year results. Surg Obes Relat Dis. 2016;12:240-5.

48. Parmar CD, Gan J, Stier C, et al. One anastomosis/mini gastric bypass (OAGB-MGB) as revisional bariatric surgery after failed primary adjustable gastric band (LAGB) and sleeve gastrectomy (SG): a systematic review of 1075 patients. Int J Surg Lond Engl. 2020;29(81):32-8.

49. Aarts E, Koehestanie P, Dogan K, et al. Revisional surgery after failed gastric banding: results of one-stage conversion to RYGB in 195 patients. Surg Obes Relat Dis. 2014;10:1077-83.

50. Carandina S, Maldonado PS, Tabbara M, et al. Two-step conversion surgery after failed laparoscopic adjustable gastric banding. Comparison between laparoscopic Roux-en-Y gastric bypass and laparoscopic gastric sleeve. Surg Obes Relat Dis. 2014;10:1085-91.

51. Lessing Y, Nevo N, Pencovich N, et al. One anastomosis gastric bypass as a revisional procedure after failed laparoscopic adjustable gastric banding. Obes Surg. 2020;30:3296-300.

52. Puzziferri N, Roshek TB, Mayo HG, et al. Long-term follow-up after bariatric surgery: a systematic review. JAMA. 2014;312:934-42.

Publisher's Note Springer Nature remains neutral with regard to jurisdictional claims in published maps and institutional affiliations. 


\section{Authors and Affiliations}

\section{Niccolò Petrucciani ${ }^{1}$ - Francesco Martini ${ }^{2}$ - Marine Benois ${ }^{3}$ - Radwan Kassir ${ }^{3} \cdot$ Hubert Boudrie $^{2}$. \\ Olivier Van Haverbeke ${ }^{2}$. Celine Hamid ${ }^{2}$. Gildas Juglard ${ }^{2}$. Gianluca Costa ${ }^{4} \cdot$ Tarek Debs $^{5}$. Arnaud Liagre ${ }^{2}$}

Francesco Martini

framartini77@gmail.com

Marine Benois

marinebenois@gmail.com

Radwan Kassir

radwankassir42@hotmail.fr

Hubert Boudrie

hboudrie@capio.fr

Olivier Van Haverbeke

drvanhaverbeke@hotmail.fr

Celine Hamid

hamidceline@gmail.com

Gildas Juglard

gildas.juglard@hotmail.fr

Gianluca Costa

gianluca.costa@uniroma1.it

Tarek Debs

debs.t@chu-nice.fr
Arnaud Liagre

arnaud.liagre@orange.fr

1 Department of Medical and Surgical Sciences and Translational Medicine, Faculty of Medicine and Psychology, St Andrea Hospital, Sapienza University, via di Grottarossa 1035-9, 00189 Rome, Italy

2 Bariatric Surgery Unit, Clinique des Cedres, Ramsay Générale de Santé, Cornebarrieu, France

3 Department of Digestive Surgery, CHU Félix Guyon, Saint Denis, La Réunion, France

4 Division of General Surgery, Campus Bio-Medico Hospital, Rome, Italy

5 Division of Digestive Surgery and Liver Transplantation, Archet II Hospital, University of Nice-Sophia-Antipolis, Nice, France 